
\title{
28 Research Square \\ The effect of warm contrast medium and other factors in pain assessment of infertile patients during HSG procedure
}

Burak Ersak ( $\sim$ burakersak@gmail.com )

Ankara City Hospital: Ankara Sehir Hastanesi https://orcid.org/0000-0003-3301-062X

Yasemin Tasci

Dumlupınar University Kutahya

Rahime Bedir Findik

University of Health Science

Duygu Tugrul

University of Health Science

Aytekin Tokmak

University of Health Science

Mahmut Kuntay Kokanali

University of Health Science

\section{Research Article}

Keywords: Hysterosalpingography, iodinated contrast media, pelvic pain warm contrast media, visual analogue scale

Posted Date: June 28th, 2021

DOl: https://doi.org/10.21203/rs.3.rs-455869/v1

License: (9) This work is licensed under a Creative Commons Attribution 4.0 International License. Read Full License 


\section{Abstract \\ Purpose}

To compare pain scores of infertile patients during and after hysterosalpingography (HSG) procedure using visual analoge scale (VAS) between cold and warm contrast medium and to investigate whether using warm contrast can alter spontaneous pregnancy rate.

\section{Methods}

Prospective randomized study between March-September 2017. Primary infertile patients undergoing HSG procedure. Patients' age, body mass index(BMI), history of previous surgery, duration of infertility and pain levels during the most painful menstrual period were recorded. The pain severity was questioned during and at the 30th minute after the procedure using VAS. While patients who underwent HSG using a contrast medium at body temperature $37^{\circ} \mathrm{C}$ were defined as warm group(n:55), patients who underwent $\mathrm{HSG}$ using a contrast medium at room temperature $23^{\circ} \mathrm{C}$ were defined as cold group(n:55).

\section{Results}

There were no significant differences between the groups in terms of age, BMI, duration of infertility, previous surgery and VAS during menstruation. The mean duration of HSG of warm group patients was significantly lower $(p=0.001)$. Warm group patients' mean VAS score during and 30 minutes after HSG were significantly lower than in the control group ( $5.3 \pm 4.2$ vs $7.5 \pm 2.0),(1.1 \pm 0.9$ vs $2.4 \pm 1.4$ ), respectively. While severe pain percentage during HSG in warm group was $\% 25.5$, it was $\% 66.7$ in the control group. In logistic regression analysis, warm and cold groups were only found as significant related factors for severe pain felt during procedure $(\mathrm{p}<0.001, \mathrm{OR}=0.19,95 \% \mathrm{Cl}=0.08-0.46)$.

\section{Conclusion}

The use of contrast media at body temperature during HSG procedure reduces the pain associated with the procedure.

\section{Introduction}

Hysterosalpingography (HSG) is an important procedure used to evaluate the intrauterine pathology, tubal lumina and patency as an initial workup of infertility[1, 2]. Although it is considered as a minimal invasive procedure, it is not easy in terms of applicability to patients. Many side effects of HSG have been reported in the literature. However, the major patient complaint of HSG is pain. The studies carried out have shown that up to $\% 85$ of patients felt pain while undergoing HSG [3]. 
Reducing or preventing the pain during the process provides better radiological outcomes and information. For this reason, prophylactic analgesia, pretreatment with corticosteroids, showing preprocedure information video were used to reduce frequency of vasavagal and pain symptoms $[4,5,6]$. Nevertheless, benefits of all these techniques are controversial; it was reported that there was no evidence of effect for oral opioid or non-steroidal anti-inflamatory drugs (NSAID) analgesia in reducing pain during the procedure or more than 30 minutes after procedure [7]. In addition, no difference in terms of pain was shown between oil- and water-soluble contrast media [8].

Warming contrast media is another intervention to reduce pain without significant risk. Studies demonstrating that using warm methods were useful for relieving pain and increasing patient comfort were limited to laparoscopic operations, peritoneal dialysis and histerocontrastsonography $[9,10,11]$ except one randomized prospective study, which has shown that warm contrast reduced visual analog scale (VAS) pain scores during the HSG procedure by alleviating peritoneal irritation [12].

HSG procedure may increase the chance to concieve due to mechanical lavage effect on the tubes and peritubal adhesions. However, the effect of using warm contrast in HSG on infertility and spontaneous pregnancy rate is unknown.

In our current study, our primary outcome was to compare pain scores with using VAS between cold and warm contrast medium. Secondary, we aimed to investigate whether using warm contrast can alter spontaneous pregnancy rate in early period.

\section{Methods}

This prospective randomized study was approved by institutional ethic committee (17.03.2017/44) and verbal/written informed consent was obtained from each participant before the enrollment.

Between March 2017 and September 2017, a total of 110 primary infertile patients without any known cause, between the ages of 19-30, who applied to Department of the Infertility and Gynecological Endocrinology Clinic, University of Health Science Zekai Tahir Burak Women's Health Education and Research Hospital were included in the study.

Patients who had acute low reproductive tract infection, genital bleeding, malignancy and known sensitivity to iodine were excluded. And also, ultrasonography was used to evaluate the ovaries in transverse and longitudinal planes. In the presence of less than 5-7 antral follicles or a FSH level $>8 \mathrm{IU} / \mathrm{L}$ were accepted as abnormal ovarian reserve test. These patients and male factor infertility were excluded from the study, as well.

For each patient, age, body mass index (BMI), history of previous pelvic infections or surgery, duration of infertility and pain levels during the most painful menstrual period within the last year were recorded. A 10-point visual analog scale (VAS) in which "0-point" was defined as painless and "10-point" as severe pain was used to assess pain severity. 
Patients were randomized into two groups according to their appointment numbers. Odd numbers constituted the 'cold group', while even numbers were included in the 'warm group'. In both groups, HSG was scheduled on days 6 through 11 from last menstrual period to avoid both the menstrual bleeding and pregnancy. HSG procedure was performed by the same gynecologist (B.E) according to Baramki's description [13] using a real-time fluoroscopy system (Zen-5000; Genoray, Korea). During the procedure, all the steps were explained to the patients. The contrast medium was stored in a room at $23^{\circ} \mathrm{C}$ normally. The cold group was composed of women who underwent HSG using a contrast medium at room temperature $23^{\circ} \mathrm{C}$, while the warm group was composed of women who underwent HSG using a contrast medium at body temperature $37^{\circ} \mathrm{C}$. The contrast medium was heated up to $37^{\circ} \mathrm{C}$ using a water bath method. Then, the temperature was confirmed using an electronic thermometer by the nurse before the injection. After the insertion of Rubin's cannula, $10 \mathrm{~mL}$ of warm or cold nonionic water-soluble contrast media (Urografin \%76 100mL, Bayer Turk Chemistry Corp.) was injected slowly into the uterine cavity while the necessary images were obtained. The gynecologist did not know which patients were given warm or cold contrast media while performing the procedure. None of the patients were given prophylactic NSAID or a spasmolytic drug to reduce pain before or during the procedure.

When the contrast media was seen passing through the fimbrial ends of the tubes, the pain severity of the patients was questioned by the gynecologist (B.E). At the 30th minute after the procedure, the presence / severity of the pain that the patient felt about the current condition was again questioned by a different physician (D.T.E) who also did not know which patients were given warm or cold contrast media. VAS was used in all these pain assessments. Patients with VAS score $\geq 6$ were accepted as severe pain. Due to the possibility of spontaneous pregnancy after HSG procedure, all cases were followed up spontaneously for 4 months and the results were recorded as a secondary objective.

Statistical analyses were carried out by using the statistical packages for SPSS 17.0 for Windows (SPSS Inc., Chicago, IL, USA). Kolmogorov-Smirnov test was used to determine the data distributions. Continuous variables were expressed as mean \pm standard deviation (SD), and categorical variables were given as number and percentage. Continuous variables were analyzed using Students' t-test and categorical variables were compared using a $\chi 2$ test. Pearson's correlation coefficient was used to analyze the correlations between VAS score during HSG and 30 minutes after HSG with other factors. Factors classified as related to severe pain felt during HSG were assessed using logistic regression model. The $p$ values less than 0.05 were considered statistically significant.

\section{Results}

During the study period, 110 patients met the inclusion criteria and participated in the study. However 4 patients of the control group were excluded due to more than $10 \mathrm{cc}$ contrast agent usage. While, 55 patients in the study group (warm group) were applied contrast agent warmed to body temperature, 51 patients were applied contrast agent at room temperature (cold group). 
There were no significant differences between the groups in terms of age, BMI, duration of infertility, previous surgery, systemic disease and VAS during menstruation. However, the mean duration of HSG of patients who were applied contrast agent warmed to body temperature was significantly lower (3.7 \pm 1.2$)$ than the mean duration of HSG $(4.4 \pm 1.2)$ of patients who were applied contrast agent at room temperature $(p=0.001)$. Furthermore, warm group patients' mean VAS score during HSG and 30 minutes after HSG were significantly lower than in the control group ( $5.3 \pm 4.2$ vs $7.5 \pm 2.0)$, ( $1.1 \pm 0.9$ vs $2.4 \pm 1.4$ ), respectively. And also, while severe pain percentage during HSG in warm group was \% 25.5 , it was $\% 66.7$ in the control group $(p<0.001)$ (Table 1$)$.

Table 1

Characteristics and pain scores of the groups

\begin{tabular}{|c|c|c|c|}
\hline & $\begin{array}{l}\text { Warm Group } \\
(n=55)\end{array}$ & $\begin{array}{l}\text { Cold Group } \\
(n=51)\end{array}$ & $\mathbf{p}$ \\
\hline Age (year) & $26.6 \pm 3.0$ & $26.3 \pm 3.3$ & 0.735 \\
\hline BMI (kg/m2) & $25,4 \pm 4,5$ & $25.5 \pm 5.7$ & 0.934 \\
\hline Duration of infertility (year) & $2.8 \pm 2.6$ & $2.3 \pm 1.5$ & 0.941 \\
\hline Previous surgery & $6(10.9)$ & $7(13.8)$ & 0.885 \\
\hline Systemic disease & $4(7.3)$ & $8(15.7)$ & 0.225 \\
\hline VAS during menstruation & $4.7 \pm 1.9$ & $5.02 \pm 1.4$ & 0.300 \\
\hline Duration of HSG (min) & $3.7 \pm 1.2$ & $4.4 \pm 1.2$ & 0.001 \\
\hline Tubal oclusion & $1(1.8)$ & $1(2.0)$ & 0.957 \\
\hline VAS score during HSG & $5.3 \pm 4.2$ & $7.5 \pm 2.0$ & $<0.001$ \\
\hline VAS score 30 minutes after HSG & $1.1 \pm 0.9$ & $2.4 \pm 1.4$ & $<0.001$ \\
\hline Severe pain during HSG & $14(25.5)$ & $34(66.7)$ & $<0.001$ \\
\hline Spontaneous pregnancy & $4(7.3)$ & $0(0.0)$ & - \\
\hline \multicolumn{4}{|c|}{ Data are shown as mean \pm standard deviation and number (\%). } \\
\hline \multicolumn{4}{|c|}{ BMI: Body mass index, VAS: Visual analog scale, HSG: Hysterosalpingography } \\
\hline
\end{tabular}

In Table 2, correlations between VAS score during HSG and 30 minutes after HSG with other factors were listed. There were no correlation between patient age, BMI, duration of infertility, pain score during menstruation and VAS score during HSG and 30 minutes after procedure. However, duration of HSG ( $\mathrm{r}=$ $0.255, p=0.008)$ and VAS score 30 minutes after HSG $(r=0.588, p<0.001)$ were positively correlated with VAS score during HSG. On the other hand, no correlation was found between duration of HSG and VAS score 30 minutes after HSG. 
Table 2

Correlations between VAS score during HSG and 30 minutes after HSG with other factors

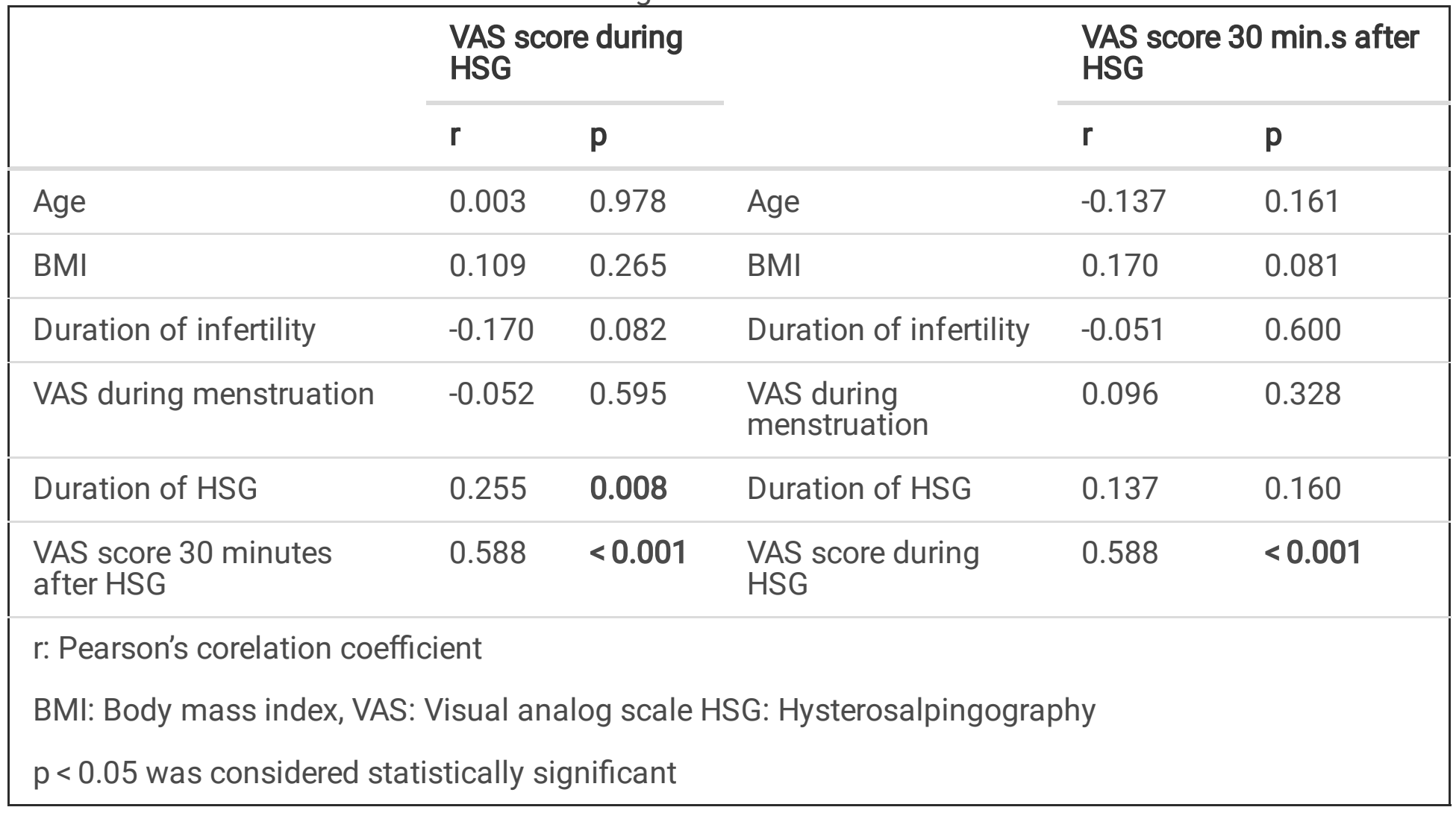

The comparison of related factors between severe and non severe pain groups are shown in Table 3. The mean duration of HSG was significantly higher in severe pain group compared with control group $(p=$ 0.034). Nevertheless, the age, BMI, duration of infertility, previous surgery, systemic disease and VAS during menstruation did not differ significantly between the groups. In logistic regression analysis, the variables cathegorized as related factors for severe pain felt during procedure were evaluated. Warm and cold groups were only found as a significant factor $(p<0.001, \mathrm{OR}=0.19,95 \% \mathrm{Cl}=0.08-0.46)$. 
Table 3

Comparison of related factors between severe and non severe pain groups

\begin{tabular}{|c|c|c|c|c|c|}
\hline & $\begin{array}{l}\text { Severe pain } \\
\text { group } \\
(\mathrm{N}=48)\end{array}$ & $\begin{array}{l}\text { Non-severe pain group ( } \mathrm{N} \\
=58)\end{array}$ & $\mathbf{P}$ & $\begin{array}{l}\text { OR } \\
(95 \% \text { C }\end{array}$ & p \\
\hline Age (year) & $26.48 \pm 3.14$ & $26.40 \pm 3.10$ & 0.892 & - & - \\
\hline $\mathrm{BMI}(\mathrm{kg} / \mathrm{m} 2)$ & $25.67 \pm 5.98$ & $25.23 \pm 4.25$ & 0.662 & - & -- \\
\hline $\begin{array}{l}\text { Duration of infertility } \\
\text { (year) }\end{array}$ & $2.33 \pm 1.67$ & $2.82 \pm 1.45$ & 0.247 & - & -- \\
\hline Previous surgery & $6(12.5)$ & $7(12.1)$ & 0.946 & - & -- \\
\hline Systemic disease & $4(8.3)$ & $8(13.8)$ & 0.377 & - & -- \\
\hline $\begin{array}{l}\text { VAS during } \\
\text { menstruation }\end{array}$ & $4.81 \pm 1.59$ & $4.88 \pm 1.72$ & 0.838 & - & - \\
\hline Duration of HSG (min) & $4.32 \pm 1.31$ & $3.80 \pm 1.19$ & 0.034 & $\begin{array}{l}1.19 \\
(0.84- \\
1.69)\end{array}$ & 0.336 \\
\hline $\begin{array}{l}\text { Groups } \\
\text { Warm } \\
\text { Cold }\end{array}$ & $\begin{array}{l}14(29.2) \\
34(70.8)\end{array}$ & $\begin{array}{l}41(70.7) \\
17(29.3)\end{array}$ & ¿ 0.001 & $\begin{array}{l}(0.08- \\
0.46)\end{array}$ & ¿. 001 \\
\hline \multicolumn{6}{|c|}{$\begin{array}{l}\text { BMI: Body mass index, VAS: Visual analog scale, HSG: Hysterosalpingography, OR: Odds ratio, Cl: } \\
\text { Confidence Interval }\end{array}$} \\
\hline
\end{tabular}

\section{Discussion}

In our study for pain that patients feel during and after HSG procedure, we reached some findings that we thought were important. Namely, when we used warm medium we found that patients felt less pain both during and 30 minute after the procedure and the presence of severe pain was more in the cold medium group. Pain felt during the procedure increased as the duration of the procedure increased and this pain was positively associated with the pain felt at 30 minutes after the procedure. However, there was no relationship between the duration of the procedure and the pain felt after the procedure. On the other hand, those who had severe pain during the procedure had a longer procedure time and less frequent use of warm medium. However, in the presence of these two factors, only the medium used was a significant factor for the presence of severe pain. 
Pain during the HSG procedure is a troublesome situation for the patient and clinician [14]. The patient may not want to have this procedure done or completed due to this pain. In addition, the clinician can be prevented from reaching an accurate result [15]. Two important mechanisms proposed in the formation of pain that occurs during the HSG procedure are, increased contractions created in the uterus and peritoneal irritation $[16,17]$. Increased prostaglandin release in the uterus or the inflammatory response developed due to irritation formed in the peritoneum may cause pain associated with this procedure [18]. Cold stimulation is a factor that increases contraction in the uterus and peritoneal sensitivity [19]. Therefore, in the presence of a cold stimulus, the patient will be expected to experience more pain. Indeed, in 2008 , two meta-analyzes by Sammour et al and Sajid et al reported that insufflation with heated $\mathrm{CO} 2$ was more advantageous in terms of postoperative pain and analgesia needs [9, 20]. In another more recent meta-analysis by Balayssac et al. it has been reported that warm insufflation is more beneficial for immediate postoperative pain [21]. On the other hand, studies on using warm medium during HSG are limited. To our knowledge, our study is the second study comparing warm and cold contrast agent for HSG. First study was published by Zhu et al in 2012 [12]. In that study, similar to ours, patients' VAS pain scores during HSG was found to be significantly lower in the warm contrast agent applied group. However, VAS pain scores at 30 minutes after procedure showed no statistical difference. In addition, we found the frequency of severe pain more in the cold group. In our study, the duration of the procedure in the cold group was longer, and the prolongation of this period increased the severity of pain felt during the procedure, and the increased pain during the procedure also increased the pain felt at 30 minutes after the procedure. Extending the duration of the HSG procedure indicates that the procedure is more difficult. This may cause more pain to be felt. As a matter of fact, we found that the duration of the procedure in patients with severe pain was longer than the group without severe pain. Although this can be seen as a factor for the presence of severe pain, we have seen that this negative effect disappears with the use of warm medium in the regression model. We think that this finding shows that the warm medium used during HSG procedure is a very effective factor in reducing HSG-related pain.

A relatively increase in spontaneous pregnancies can be observed after HSG procedure, and a number of theories are proposed as a reason [22]. Tubal flushing during HSG may increase the chance of pregnancy [23]. Depending on the contrast type used, endometrial receptivity and implantation rate may increase [24]. Sperm phagocytosis can be prevented due to the immunological response in the peritoneal cavity, and tubal ciliary activity may also increase $[25,26]$. In our study, 4 spontaneous pregnancies were detected in the warm group, but no pregnancy occurred in the cold group during 4-month follow-up period after HSG. This suggests that warm medium may be more beneficial for pregnancy occurrence. However, this finding is a new information for the literature and it should be supported with additional studies with wider participation.

The fact that it is a randomized study on a subject that has not been studied much and that it contains new findings for the literature is the strong features of our study. In addition, the homogeneous groups and the HSG process being performed by the same person and the feature of the contrast material given are not known by the person performing the procedure, provides a more reliable comparison of the results. On the other hand, the relatively small number of participants and the infertile group with certain 
characteristics may create limitations in generalizing our findings for all infertile patients. In addition, the psychological state of the individuals before the procedure, for example anxiety levels, may affect the level of HSG related pain. It is a limitation that such factors are not evaluated in our study.

As a result, the use of contrast media at body temperature during HSG procedure reduces the pain associated with the procedure. It may also increase the chance of spontaneous pregnancy after the procedure. Nevertheless, additional prospective randomized trials with more participation are needed to achieve the accuracy of such beneficial results in clinical practice.

\section{Declarations}

\section{Funding}

No funding was received for the study.

\section{Compliance with Ethical Standards}

All procedures performed were in accordance with the ethical standarts of the institutional committee (17.03.2017/44) and with the 1964 Helsinki declaration and its later amendments or comparable ethical standards.

\section{Conflict of interest}

The authors declare that they have no conflict of interest.

The authors declare no financial disclosure.

All authors read and approved the final manuscript.

This manuscript is not under consideration for publication anywhere else.

\section{Acknowledgements}

The authors declare no conflicts of interest.

\section{Disclosure}

None declared

\section{Informed consent}

Informed consent was obtained from all individual participants included in the study

\section{Authors contributions}

B Ersak: Data collection, design, manuscript writing 
Y Tasci: Project development

RB Findik: Concept

D Tugrul: Data collection, literature search

A Tokmak: Analysis, interpretation

MK Kokanali: Critical reviews, manuscript editing

All authors read and approved the final version of the manuscript.

\section{References}

1. Liberty G, Gal M, Halevy-Shalem T et al (2007) Lidocaine-Prilocaine (EMLA) cream as analgesia for hysterosalpingography: a prospective, randomized, controlled, double blinded study. Hum Reprod 22(5):1335-1339. https://doi.org/10.1093/humrep/del517

2. Roma Dalfó $A$, Úbeda $B$, Úbeda $A$ et al (2004) Diagnostic value of hysterosalpingography in the detection of intrauterine abnormalities: a comparison with hysteroscopy. Am J Roentgenol 183(5):1405-1409. https://doi.org/10.2214/ajr.183.5.1831405

3. Costello MF, Horrowitz S, Steigrad S et al (2002) Transcervical intrauterine topical local anesthetic at hysterosalpingography: a prospective, randomized, double-blind, placebo-controlled trial. Fertil Steril 78(5):1116-1122. https://doi.org/10.1016/s0015-0282(02)03362-9

4. Maddox TG (2002) Adverse Reactions to Contrast Material. Recognition, Prevention and Treatment. Am Fam Physician 66(7):1229

5. Shalev J, Krissi H, Blankstein J et al (2000) Modified hysterosalpingography during infertility workup: use of contrast medium and saline to investigate mechanical factors. Fertil Steril 74(2):372-375. https://doi.org/10.1016/s0015-0282(00)00632-4

6. Erkılınç S, Kala NA, Pekcan MK et al (2018) The effect of a pre-procedure information video on anxiety levels in patients undergoing hysterosalpingography: A prospective case-control study. Journal of the Turkish German Gynecological Association 19(3):137. https://doi.org/10.4274/jtgga.2017.0118

7. Ahmad G, Duffy JM, O'Flynn H et al. Pain relief in hysterosalpingography. Cochrane Database Syst Rev. 2007 (2). https://doi.org/10.1002/14651858.CD007710.pub2

8. Lindequist S, Justesen P, Larsen C et al (1991) Diagnostic quality and complications of hysterosalpingography: oil-versus water-soluble contrast media-a randomized prospective study. Radiology 179(1):69-74. https://doi.org/10.1148/radiology.179.1.1848715

9. Sammour T, Kahokehr A, Hill A (2008) Meta-analysis of the effect of warm humidified insufflation on pain after laparoscopy. Br J Surg 95(8):950-956. https://doi.org/10.1002/bjs.6304 
10. Zheng Z-H, Anderstam B, Qureshi AR et al (2002) Heat sterilization of peritoneal dialysis solutions influences ingestive behavior in non-uremic rats. Kidney Int 62(4):1447-1453. https://doi.org/10.1111/j.1523-1755.2002.kid575.x

11. Nirmal D, Griffiths A, Jose $G$ et al (2006) Warming Echovist contrast medium for hysterocontrastsonography and the effect on the incidence of pelvic pain. A randomized controlled study. Hum Reprod 21(4):1052-1054. https://doi.org/10.1093/humrep/dei440

12. Zhu Y-Y, Mao Y-Z, Wu W-L (2012) Comparison of warm and cold contrast media for hysterosalpingography: a prospective, randomized study. Fertil Steril 97(6):1405-1409. https://doi.org/10.1016/j.fertnstert.2012.02.039

13. Baramki TA. Hysterosalpingography (2005) Fertil Steril 83(6):1595-1606. https://doi.org/10.1016/j.fertnstert.2004.12.050

14. Chauhan MB, Lakra P, Jyotsna D et al (2013) Pain relief during hysterosalpingography: role of intracervical block. Arch Gynecol Obstet 287(1):155-159. https://doi.org/10.1007/s00404-012-2515z

15. Horwitz R, Morton P, Shaff M et al (1979) A radiological approach to infertilityhysterosalpingography. Br J Radiol 52(616):255-262. https://doi.org/10.1259/0007-1285-52-616255

16. Safi F, Kamali A, Rezaei M et al (2019) Effect of intramuscular hyoscine-n-butyl bromide on fallopian tube spasm and pain perception during and after hysterosalpingography in infertile women: $\mathrm{A}$ randomized single-blind controlled clinical trial. Med J Islam Repub Iran 33:31. https://doi.org/10.34171/mjiri.33.31

17. Hindocha A, Beere L, O'Flynn $\mathrm{H}$ et al. Pain relief in hysterosalpingography. Cochrane Database Syst Rev. 2015 (9). https://doi.org/10.1002/14651858.CD006106.pub3

18. Gupta N, Ghosh B, Mittal S (2008) Comparison of oral naproxen and intrauterine lignocaine instillation for pain relief during hysterosalpingography. International Journal of Gynecology Obstetrics 102(3):284-286. https://doi.org/10.1016/j.ijgo.2008.04.013

19. Wang $D$, Cheng $X$, Fang $H$ et al (2020) Effect of cold stress on ovarian \& uterine microcirculation in rats and the role of endothelin system. Reprod Biol Endocrinol 18:1-13. https://doi.org/10.1186/s12958-020-00584-1

20. Sajid MS, Mallick AS, Rimpel J et al (2008) Effect of heated and humidified carbon dioxide on patients after laparoscopic procedures: a meta-analysis. Surgical Laparoscopy Endoscopy Percutaneous Techniques 18(6):539-546. https://doi.org/10.1097/SLE.0b013e3181886ff4

21. Balayssac D, Pereira B, Bazin J-E et al (2017) Warmed and humidified carbon dioxide for abdominal laparoscopic surgery: meta-analysis of the current literature. Surg Endosc 31(1):1-12. https://doi.org/10.1007/s00464-016-4866-1

22. Van Welie N, Dreyer K, Van Rijswijk J et al (2019) Treatment effect of oil-based contrast is related to experienced pain at HSG: a post-hoc analysis of the randomised H2Oil study. Hum Reprod 34(12):2391-2398. https://doi.org/10.1093/humrep/dez206

Page $11 / 12$ 
23. Dreyer K, van Eekelen R, Tjon-Kon-Fat R et al (2019) The therapeutic effect of hysterosalpingography in couples with unexplained subfertility: a post-hoc analysis of a prospective multi-centre cohort study. Reprod Biomed Online 38(2):233-239. https://doi.org/10.1016/j.rbmo.2018.11.005

24. Johnson NP (2014) Review of lipiodol treatment for infertility-an innovative treatment for endometriosis-related infertility? Aust N. Z J Obstet Gynaecol 54(1):9-12. https://doi.org/10.1111/ajo.12141

25. Izumi G, Koga K, Takamura M et al (2017) Oil-soluble contrast medium (OSCM) for hysterosalpingography modulates dendritic cell and regulatory $\mathrm{T}$ cell profiles in the peritoneal cavity: a possible mechanism by which OSCM enhances fertility. J Immunol 198(11):4277-4284. https://doi.org/10.4049/jimmunol.1600498

26. Mohiyiddeen L, Hardiman A, Fitzgerald C et al (2015 (5)) Tubal flushing for subfertility. Cochrane Database Syst Rev. https://doi.org/10.1002/14651858.CD003718.pub4 